\title{
FAST MODEL BASED SEGMENTATION OF ULTRASOUND DATA USING AN ACTIVE IMAGE
}

\author{
Dieter Hönigmann ${ }^{a}$, Johannes Ruisz ${ }^{a}$, Helmut Pottmann ${ }^{b}$ \\ ${ }^{a}$ Advanced Computer Vision GmbH - ACV, Vienna, Austria \\ ${ }^{b}$ Institute of Geometry, Vienna University of Technology, Vienna, Austria
}

\begin{abstract}
In the present paper we propose a method for fast segmentation of ultrasound data. It is based on setting up a model depending on user input. We apply a matching scheme in order to obtain initial contours for 2D segmentation of several cross-sections of the organ by a discrete dynamic contour. Further, we set up an active image which drives the deformation of the dynamic contour. The active image comprises both textural information based on image data as well as spatial information which we derive from the inital contour. We design the active image according to user input and image quality to aid the segmentation task.
\end{abstract}

\section{INTRODUCTION}

Currently, ultrasound imaging is in need of fast methods capable of segmenting data sets. Subsequently we will present a framework capable of performing this task.

Numerous approaches have been proposed to segment ultrasound data by means of deformable models. Some recent works are those of Lefebvre et al. [1], Shekhar et al. [2] and Chen et al. [3]. Unfortunately, application of these methods to large data sets arising from 3D data acquisition is either compuationally demanding or restricted to data of particular nature, e.g. acquired by intravascular ultrasound imaging.

Our scheme is based upon discrete dynamic contours (DDC) applied to cross sections of the organ. The main advantage of the DDC approach is its conceptual and computational simplicity while taking into account both image features and spatial information. On the other hand, there are properties of DDCs which make their application to ultrasound data somewhat difficult. First, the method is sensitive to the image features which drive the deformation of the contour. Second, accurracy and speed of convergence to the desired final contour depend on a reasonable initial contour.

This work had been carried out within the K plus Competence Center ADVANCED COMPUTER VISION. This work was funded from the K plus Program.
We provide a solution to these issues which can be implemented in an efficient way. It is the key idea of our approach to base the DDC segmentation on an active image, comprising a spatial and a textural component. In order to set up this image we first compute a rough estimate of an initial contour.

\section{INITIAL CONTOUR}

The scheme we propose requires the acquisition of several 2D images obtained by rotating the image plane about an axis $\mathbf{a}_{\text {rot }}$. The position of $\mathbf{a}_{\text {rot }}$ has to be chosen in allignment with a reference axis given together with a 3D model of the organ. We define the position of the axis by two points which also delimit the extent of the organ along the axis. We can now obtain an accurrate initial contour $C_{\text {init }}$ by including a-priori information about the approximate shape of the organ as follows:

We construct a set $M$ of $2 \mathrm{D}$ masks $m_{\mathrm{i}}$ by computing cross-sections of the model of the organ with a plane that rotates about $\mathbf{a}_{\text {rot }}$. For every mask $m_{\mathrm{i}} \in M$ we compare textural properties of the image region covered by $m_{\mathrm{i}}$ with textural properties of a reference area $m_{\text {ref }}$. We set up the reference area in a way which guarantees that it is located within the image of the organ. We assess textural similarity by means of parameters computed by evaluation of cooccurrence matrices as proposed by Valckx et al. in [4]. The result of texture evaluation is a single feature vector $\mathbf{f}_{\mathrm{i}}$ for every mask $m_{\mathrm{i}}$ and a feature vector $\mathbf{f}_{\mathrm{ref}}$ for the reference mask. We attain this similarity quantitatively by normalizing $\mathbf{f}_{\mathrm{i}}$ and $\mathbf{f}_{\mathrm{ref}}$ according to a suggestion of Aksoy and Haralick in [5] and calculating the sum of absolute differences between the vector $\mathbf{f}_{\text {ref }}$ and all vectors $\mathbf{f}_{\mathrm{i}}$. Further, our scheme takes into account that large masks tend to be more different to a relatively small reference area than smaller masks. Therefore, we utilize scaling that favours large masks while imposing a "penalty" on smaller ones. Finally, we yield a score for every mask $m_{\mathrm{i}}$ indicating its textural similarity with respect to $m_{\text {ref }}$.

After selecting an appropriate mask $m_{\mathrm{i}}$ according to this scheme we draw a number of points $V$ from the perimeter. 
These points are the vertices of the initial contour $C_{\text {init }}$.

\section{ACTIVE IMAGE}

As lined out in section 1 we aim at an active image that is based upon the ultrasound image but adapts to the segmentation task according to user input and image quality. We construct the active image $I_{\mathrm{A}}$ from a spatial component $I_{\mathrm{S}}$ and a textural component $I_{\mathrm{T}}$. We design $I_{\mathrm{A}}$ in a way that ensures that pixels with a high probability of being within the image of the organ obtain low values. Pixels with high values are most probably outside the image of the organ under consideration.

\subsection{Spatial Component}

Having obtained the initial contour $C_{\text {init }}$ according to section 2 we can easily obtain the interior $R_{\text {init }}$ of $C_{\text {init }}$. The computation of $I_{\mathrm{S}}$ is based upon the idea to compute the shortest distance of every pixel $(x, y)$ to $R_{\text {init }}$. For our application it is sufficient to compute an approximation of this function as follows. The graph of the Euclidean distance of every point $(x, y)$ of a plane to a fixed point $V$ is a cone. We obtain the graph of the distance function to a different point $V^{\prime}$ by translation of the cone. Thus, for every position $(x, y)$ we evaluate the distance functions with respect to every vertex $V \in C_{\text {init }}$ and compute their minimum. Having obtained an estimation of the distance $d(x, y)$ to $R_{\text {init }}$ for every position $(x, y)$ we set up $I_{\mathrm{S}}$ as

$$
I_{\mathrm{S}}(x, y)= \begin{cases}\sqrt{d(x, y)} & \text { if }(x, y) \in R_{\text {init }} \\ 0 & \text { if }(x, y) \notin R_{\text {init }}\end{cases}
$$

The graph of $I_{\mathrm{S}}$ in Fig. 1 shows that the influence of every vertex $V \in C_{\text {init }}$ decreases with its square distance.

\subsection{Textural Component}

As mentioned above, generally, the sole consideration of pixel intensities is an insufficient basis to drive the deformation of a DDC. Therefore, texture analysis is required. There are several different approaches to extract texture from ultrasound data which are beyond the scope of this paper. We employ a simple and fast scheme that computes a feature vector $\mathbf{f}(x, y)$ by evaluation of the mean and variance of intensities in a small neighbourhood of every pixel $(x, y)$.

Similar to the spatial component $I_{\mathrm{S}}$ we aim at a textural component $I_{\mathrm{T}}$ with low values at pixels inside the image of the organ. Therefore, we have to introduce a proper measure of similarity between several textural reference vectors $\mathbf{f}_{\text {ref }}(x, y)$ drawn from pixels $(x, y) \in R_{\text {init }}$ and the feature vectors $\mathbf{f}(x, y)$ of all other pixels of the image. We sug-

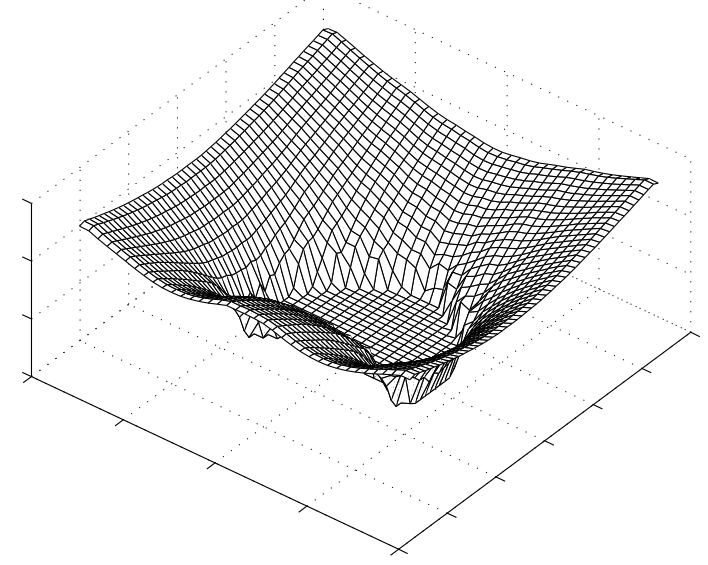

Fig. 1. Graph of the spatial component $I_{\mathrm{S}}$ of an active image.

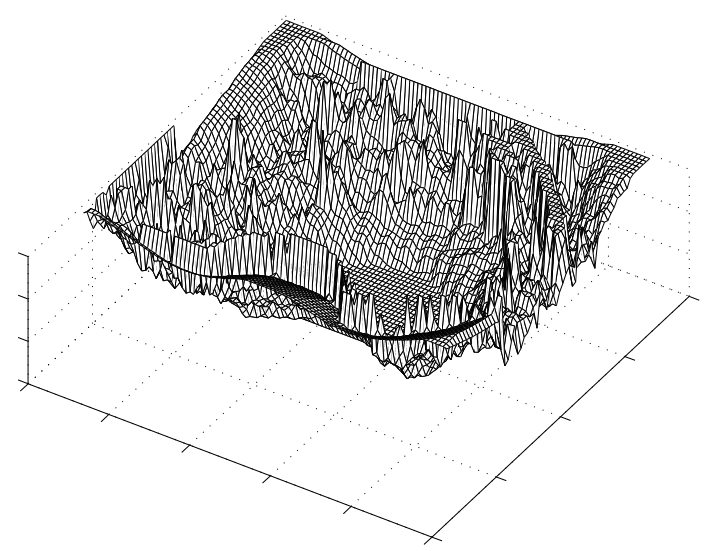

Fig. 2. Graph of the active image $I_{\mathrm{A}}$.

gest to employ the Mahalanobis metric

$$
\begin{array}{r}
I_{\mathrm{T}}(x, y)=\sqrt{\mathbf{f}(x, y) \cdot \Sigma^{-1} \cdot \mathbf{f}(x, y)^{T}}, \\
\Sigma=\operatorname{cov}\left(\left\{\mathbf{f}_{\text {ref }}(x, y)\right\}\right),
\end{array}
$$

using the covariance matrix of the reference vectors as scaling matrix.

\subsection{Setting Up the Active Image}

The active image is the result of the combination of the spatial information $I_{\mathrm{S}}$ with the textural information $I_{\mathrm{T}}$. We suggest a combination of the form

$$
I_{\mathrm{A}}=\left(I_{\mathrm{T}}+\lambda\right) \cdot I_{\mathrm{S}} .
$$

$\lambda$ is a parameter that balances the influence of $I_{\mathrm{S}}$ and $I_{\mathrm{T}}$ in the active image $I_{\mathrm{A}}$. Proper adjustment of this parameter ac- 
cording to image quality is of great importance. In images with large areas of distinctive texture we set $\lambda$ to small values in order to increase the influence of $I_{\mathrm{T}}$. If image quality is poor we set $\lambda$ to larger values to obtain an active image with stronger influence of $I_{\mathrm{S}}$.

We worked out means to determine $\lambda$ automatically by estimating image quality within $R_{\text {init }}$. The estimation is based upon the evaluation of the compactness of several threshold operations in the textural domain. An active image based on $I_{\mathrm{S}}$ in Fig. 1 is depicted in Fig. 2.

\section{DISCRETE DYNAMIC CONTOUR}

Having computed $I_{\mathrm{A}}$ we now apply a DDC segmentation which is slightly different from the usual scheme as proposed, e.g., by Lobregt and Viergever in [6]. The total force acting on every vertex is a weighted combination of an external force $\mathbf{F}_{\text {ext }}$ which drives the expansion of the DDC and an internal force $\mathbf{F}_{\text {int }}$ which provides for a smooth contour. In addition, we use a stopping term $t_{\text {stop }}$ which provides deceleration of motion outside the target region. These components are combined for every single vertex $V \in C$ according to

$$
V_{t+1}=V_{t}+t_{\text {stop }}\left(\alpha \mathbf{F}_{\text {ext }}+\beta \mathbf{F}_{\text {int }}\right) .
$$

The external force $\mathbf{F}_{\text {ext }}$ is a mere balloon force expanding the polygon in the direction of the outer bisectors of the edges adjacent to $V$. The magnitude of $\mathbf{F}_{\text {ext }}$ is determined by the constant $\alpha$.

The aim of the internal force $\mathbf{F}_{\text {int }}$ is to reduce the curvature of the polygon. We obtain an estimate of the curvature $\kappa$ at $V$ and compute

$$
\beta=1-e^{-\kappa^{2}}
$$

The factor $\beta$ is determining the magnitude of the internal force. The direction of $\mathbf{F}_{\text {int }}$ is towards the center of gravity of the triangle set up by the adjacent egdes of $V$.

Finally, the stopping term $t_{\text {stop }}$ is responsible for stopping the movement of the DDC. It is set up according to

$$
t_{\text {stop }}=\frac{1}{1+\frac{I_{\mathrm{A}} \cdot\left|\nabla I_{\mathrm{A}}\right|}{\mu^{2}}} .
$$

It incorporates the pure active image $I_{\mathrm{A}}$ as well as its gradient $\nabla I_{\mathrm{A}}$. The parameter $\mu$ allows for an adjustment of the sensitivity of the stopping term.

\section{3D MODEL BASED ON CROSS-SECTIONS}

The proposed approach allows us to obtain a 3D model of the organ under examination in a straightforward way. After an initial 2D image acquisition and segmentation we rotate the imaging plane about the axis $\mathbf{a}_{\text {rot }}$ by a small angle and obtain the segmentation of the respective image without any further interaction of the user. After subsequent rotation by $180^{\circ}$ with respect to the first image we can construct a 3Dmodel of the organ under examination by triangular meshing of the vertices of adjacent contours.

\section{RESULTS}

In the present section we show results on ultrasound data of a prostate using our active image DDC approach. In order to assess its performance we have been implementing a Matlab prototype capable of model matching, active image computation and DDC initialization and deformation. The present set of models of the prostate assumes axial symmetry of the organ. Of course, anatomically this is not true but, nevertheless, even with this very simplistic model we obtain good results.

We employ our scheme to segment cross-sections which have been extracted from a $167 \times 152 \times 238$ voxel data set of a prostate with 8 bit intensity quantization.

A subset of the masks used for estimation of the initial contour of the cross-section is depicted in Fig. 3. Below every mask its score is noted. The mask with the minimal score (0.129), indicating the most similar texture to a reference area, fits very well to the shape of the prostate in this cross-section. The mask with the second lowest score $(0.153)$ would have been another reasonable estimate.

We shrink the initial contour $C_{\text {init }}$ and draw a set of 28 vertices from its perimeter. In this way we guarantee that all vertices are located within the image of the prostate. Based

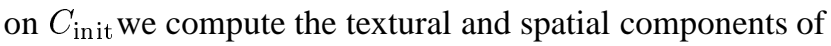
the active image and combine them in the proposed way. We start the DDC progression which stops at the position indicated in Fig. 4.

The initial contour and the result of an acquisition plane rotated by $90^{\circ}$ with respect to Fig. 4 is depicted in Fig. 5 .

\section{DISCUSSION AND OUTLOOK}

The active image is not only a good substrate to drive the deformation of a DDC. Originally, we designed the scheme to aid a 2D segmentation of ultrasound images by level set propagation. Thereby, we obtained very promising results, too. The main reason for favoring DDC segmentation is its easy extendability for fast segmentation of $3 \mathrm{D}$ data as proposed in section 5 .

Calculation of the initial contour is a crucial part of our scheme. Selecting an initial contour based on a too large mask would lead to undesired results because both the initial DDC and the active image which drives its deformation are effected. Nevertheless, our mask matching scheme performed very well in various tests. 


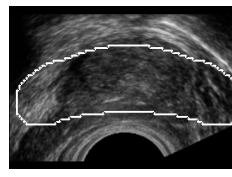

0.544

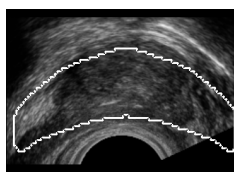

0.505

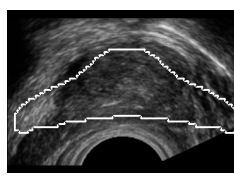

0.638

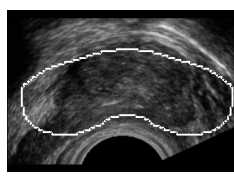

0.475

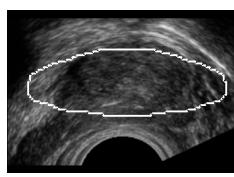

0.614

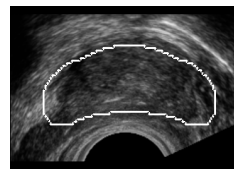

0.497

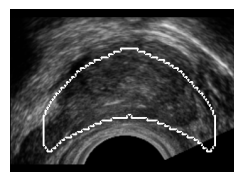

0.368

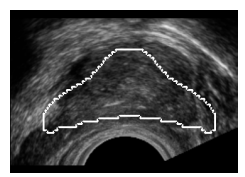

0.423

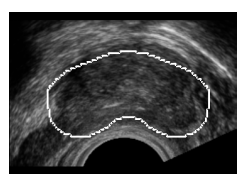

0.374

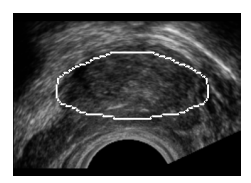

0.366

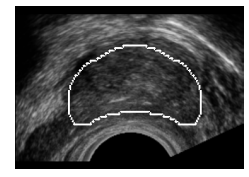

0.129

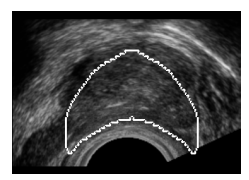

0.230

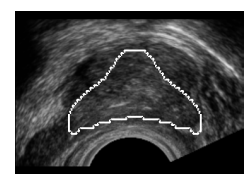

0.199

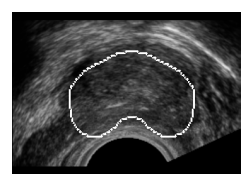

0.153

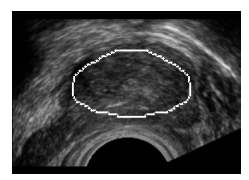

0.207
Fig. 3. Subset of masks employed to estimate the initial contour $C_{\text {init }}$. Below every mask its score is noted. The minimum score (first row, right column) belongs to the mask with most similar texture.

Future extensions of our scheme aim at a method to compute an initial contour which combines mask matching with the result of the previous DDC segmentation. In an even further extension we plan to modify the initial 3D model from which the masks are taken according to the result of two initial segmentations of orthgonal imaging planes. Then we use the refined 3D model for initial contour estimation for the final segmentation.

\section{ACKNOWLEDGMENT}

We wish to thank Armin Schoisswohl from Kretztechnik AG for valuable suggestions concerning the DDC during a very inspiring meeting in July, 2001.

\section{REFERENCES}

[1] F. Lefebvre, G. Berger, and P. Laugier, "Automatic detection of the boundary of the calcaneus from ul-

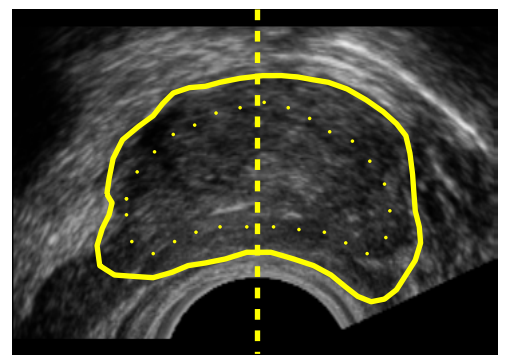

Fig. 4. Initial DDC (doted line) and result of the DDC segmentation (solid line) of the first imaging plane. The axis $\mathbf{a}_{\text {rot }}$ is indicated by the dashed line.

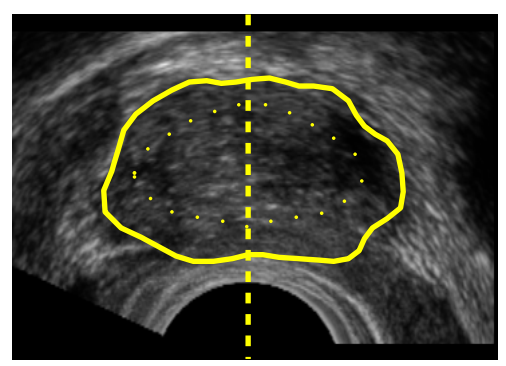

Fig. 5. Initial DDC (doted line) and result of the DDC segmentation (solid line) of the imaging plane orthogonal to Fig. 4.

trasound parametric images using an active contour model," IEEE Transactions on Medical Imaging, vol. 17, pp. 45-52, 1998.

[2] R. Shekhar, R.M. Cothren, and D.G. Vince, "Threedimensional segmentation of luminal and adventitial borders in serial intravascular ultrasound images," Comput Med Imaging Graphics, vol. 23, pp. 299-309, 1999.

[3] C.M. Chen, H.H.S. Lu, and A.T. Hsiao, "A dualsnake model of high penetrability for ultrasound image boundary extraction," Ultrasound in Medicine and Biology, vol. 27, no. 12, pp. 1651-1665, 122001.

[4] F.M.J. Valckx and J.M. Thijssen, "Characterization of echographic image texture by co-occurrence matrix parameters," Ultrasound in Medicine and Biology, vol. 23, no. 4, pp. 559-571, 1997.

[5] S. Aksoy and R.M. Haralick, "Feature normalization and likelihood-based similarity measures for image retrieval," Pattern Recognition Letters, vol. 22, pp. 563582, 2001.

[6] S. Lobregt and M.A. Viergever, "A discrete dynamic contour model," IEEE Transactions on Medical Imaging, vol. 14, pp. 12-24, 1995. 